\title{
Retrospective analysis of safety and efficacy of enhanced recovery pathways in stage II- III colorectal cancer patients submitted to surgery and adjuvant therapy
}

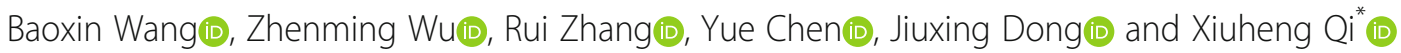

\begin{abstract}
Background: The American Society of Colon and Rectal Surgeons is suggesting laparoscopic surgeries for colorectal cancer. Conventional perioperative procedures like long preoperative fasting and bowel procedures are not useful and harmful to patients undergoing surgeries for colorectal cancer. The objectives of the study were to compare surgery outcomes, hospital stays, and survival of patients who received fast-track (laparoscopy/open) surgical procedure followed by chemotherapy against those who received conventional (laparoscopy/open) surgical procedure followed by chemotherapy for colorectal cancer.
\end{abstract}

Methods: The study analyzes the outcomes of a total of 542 colorectal cancer (preoperative biopsies stage II or III) patients submitted to surgery and adjuvant chemotherapy. The study cohort is retrospectively subdivided in 4 groups submitted to open or laparoscopic resection with or without fast-track protocol appliance and two different chemotherapy regimens. Patients who ended up being TNM stage I have not received the adjuvant chemotherapy.

Results: The fast-track surgical procedure had shorter total hospital stays and postoperative hospital stays than the conventional surgical procedures. Flatus resumption time, the time until first defecation, and intraoperative blood loss were shorter for the fast-track surgical procedures than the conventional surgical procedures. Those surgery outcomes were also shorter for the fast-track laparoscopy than the open fast-track. Resumption of a fluid diet and ambulation onset time were shorter for the fast-track surgical procedures than the conventional surgical procedures. The surgical checkpoints that were compliance by patient of fast-track surgeries were significantly fewer than those of the conventional surgeries. Clinically significant difference for QLQ-C30/CR38 score after chemotherapy was reported between patients who received open conventional surgeries and those patients who received fast-track laparoscopy (59.63 $\pm 2.26 \mathrm{score} /$ patient vs. $71.67 \pm 5.19 \mathrm{score} /$ patient). There were no significant differences for the number of patients with any grade adverse effects $(p=0.431)$ or with grade $3-4$ adverse effects $(p=0.858)$, and the disease-free and overall survival among cohorts.

Conclusions: The fast-track surgical procedure is effective and safe even in a multidisciplinary scenario as colorectal cancer treatment in which surgery is only a part of management.

Level of evidence: III: Technical efficacy stage: 4.

Keywords: Colorectal cancer, Chemotherapy, Fast-track, Hospital stay, Laparoscopy, Open surgical procedure

\footnotetext{
* Correspondence: xiuheng.qi@gmail.com
}

Department of Oncology, Hebei Petrochina Central Hospital, Heibei,

Langfang 065000, China 


\section{Background}

Colorectal cancer was the third most common cancer worldwide in 2017 [1] and the second most common cancer worldwide in 2020 [2]. Colorectal cancer is the fifth most common cancer responsible for death [3]. Patients with colorectal cancer are generally cured by radical surgery followed by chemotherapy and/or radiotherapy regimens [4]. The American Society of Colon and Rectal Surgeons is suggesting laparoscopic surgeries for colorectal cancer [5]. Conventional perioperative procedures like long preoperative fasting and bowel procedures are not useful and even harmful to patients undergoing surgeries for colorectal cancer [6, 7]. Fast-track surgery includes several perioperative interventions for the enhancement of recovery of patients [4].

LAFA-study [8] and EnROL trial [9] on the Caucasian population and the randomized trials on the Chinese population $[4,10-13]$ are reporting that the fast-track surgery is effective for colorectal cancer patients regarding postoperative recoveries. However, trials on the Chinese population [4, 10-13] are not comprehensive, some are with a small number of patients and did not report preoperative procedural details, and only 1-week postoperative period are studied. However, patients may require 6 months of chemotherapy after surgery [4]. Also, some of the surgical procedures recommended by LAFA study [8] and EnROL trial [9] are difficult to implement in the Chinese population.

Therefore, the fast-track surgical model for multidisciplinary treatment protocol [14] of the Chinese population is taken into consideration for surgeries for colorectal cancer in several institutes of China, which includes more conservative surgeries than those of the Western countries. Also, it covers the overall treatment process.

The primary aim of the retrospective study was to compare surgical checkpoints that were compliance by patients, surgery outcomes, and length of hospital stays of patients who underwent fast-track surgery (laparoscopy/open surgical procedure) against those who underwent conventional surgery (laparoscopy/open surgical procedure) for colorectal cancer. The secondary aim of the study was to compare chemotherapy-related adverse events, quality of life after chemotherapy regimens, and survival during follow-up of patients who received capecitabine and oxaliplatin chemotherapy against those who received leucovorin, fluorouracil, and oxaliplatin chemotherapy regimens after surgery for colorectal cancer.

\section{Methods}

\section{Study population}

Patients ( $>18$ years old) with pathologically confirmed colon or upper rectal (the distance of tumor lower margin from anus $>12 \mathrm{~cm}$ ) cancer (preoperative biopsies stage II or stage III) from 15 January 2018 to 25 December 2019 were included in the analysis. Patients whose tumor were removed by the endoscopic mucosal procedure(s), patients who are pregnant, patients who have a spinal deformity, and patients who had undergone surgery followed by chemotherapy of mid-low rectal cancer were excluded from the analysis, as this would have greatly affected the main outcomes of the study.

\section{Sample size calculation}

Based on patients need for adjuvant chemotherapy, power of $80 \%(\beta=0.2), 5 \%$ of two-sided type-I error $(\alpha=0.05)$, and $95 \%$ of confidence level [4], there was a need of a minimum of 500 patients with at least 125 patients in each cohort.

\section{Cohort}

A total of 125 patients were subjected to fast-track laparoscopic surgery followed by 8 cycles ( 21 days in between two cycles) of $850 \mathrm{mg}-1 \mathrm{~g} / \mathrm{m}^{2}$ capecitabine (Xeloda, F. Hoffmann-La Roche AG, Grenzacherstrasse, Basel, Switzerland) and $130 \mathrm{mg} / \mathrm{m}^{2}$ oxaliplatin (Eloxatin, Sanofi-aventis, rue La Boetie, Paris, France) chemotherapy (if required) (FTLCO cohort). A total of 137 patients were subjected to open fast-track surgery followed by 8 cycles ( 21 days in between two cycles) of $850 \mathrm{mg}-1 \mathrm{~g} / \mathrm{m}^{2}$ capecitabine and $130 \mathrm{mg} / \mathrm{m}^{2}$ oxaliplatin chemotherapy (if required) (OTLCO cohort). A total of 142 patients were subjected to conventional laparoscopic surgery followed by 12 cycles ( 15 days in between two cycles) of $400 \mathrm{mg} / \mathrm{m}^{2}$ leucovorin (Wellcovorin, Wyeth Pharmaceuticals Limited, Overland Park, Kansas, USA), $400 \mathrm{mg} / \mathrm{m}^{2}$ 5-fluorouracil (Adrucil ${ }^{\circ}$, Teva Pharamceuticals Inc., North Wales, PA, USA), and $85 \mathrm{mg} / \mathrm{m}^{2}$ oxaliplatin chemotherapy (if required) (CSLFO cohort). A total of 138 patients subjected to open conventional surgery followed by 12 cycles ( 15 days in between two cycles) of $400 \mathrm{mg} / \mathrm{m}^{2}$ leucovorin, $400 \mathrm{mg} / \mathrm{m}^{2}$ 5-fluorouracil, and $85 \mathrm{mg} / \mathrm{m}^{2}$ oxaliplatin chemotherapy (if required) (OSLFO cohort). The differences between fast-track and the conventional surgical procedures are summarized in Table 1 [14]. All surgical procedures were performed according to the National Comprehensive Cancer Network Clinical Practice Guidelines in Oncology ${ }^{\circ}$ Colon Cancer V.2.2018 [15] by colorectal surgeons (with a minimum of 3 years of institutional training of abdominal surgeries) of the institutes. The chemotherapy was given by nursing staff (minimum 3 years of experiences; aware of the type of surgeries) of the institutes. The different adjuvant therapies to the factorial design of the treatment subgroups were added because of the instruction of the institute (institutional protocol).

Data regarding surgical checkpoints, hospital stays, outcomes for surgery, chemotherapy-related adverse events, quality of life after chemotherapy, and survival 
Table 1 The differences between fast-track and the conventional surgical procedures for colorectal cancer

\begin{tabular}{|c|c|c|}
\hline Parameters & Fast-track surgical procedure & The conventional surgical procedure \\
\hline Preadmission & Mental optimism. & No mental optimism. \\
\hline \multirow[t]{3}{*}{ Counseling } & Pre-assessment for risk adjustment. & Pre-assessment for risk adjustment. \\
\hline & $\begin{array}{l}\text { Information for thoracic epidural and } \\
\text { general combined anesthesia. }\end{array}$ & No information for combined anesthesia. \\
\hline & $\begin{array}{l}\text { Information for fast-track surgical } \\
\text { procedure and consent. }\end{array}$ & $\begin{array}{l}\text { Information for the conventional surgical procedure } \\
\text { and consent. }\end{array}$ \\
\hline \multirow[t]{4}{*}{ Surgical preparation } & Bowel preparation. & Bowel preparation. \\
\hline & Enemas. & Enemas. \\
\hline & The last meal: $2 \mathrm{~h}$ before surgery. & The last meal: $10 \mathrm{~h}$ before surgery. \\
\hline & $\begin{array}{l}\text { Complete enteral nutritional. } 500 \mathrm{~mL} \\
10 \% \text { glucose } 2-3 \mathrm{~h} \text { before surgery } \\
\text { (if required). }\end{array}$ & No oral intake on the day of surgery. \\
\hline Gastrointestinal decompression & $\begin{array}{l}\sim 30 \text { min before surgery by the } \\
\text { nasogastric tube. }\end{array}$ & 30 min before surgery by the nasogastric tube. \\
\hline \multirow[t]{3}{*}{ Perioperative management } & Thoracic epidural anesthesia. & No thoracic epidural anesthesia. \\
\hline & $\begin{array}{l}\text { Balanced combination with the general } \\
\text { anesthesia. }\end{array}$ & Normal general anesthesia. \\
\hline & Mechanical ventilation. & Mechanical ventilation. \\
\hline General anesthesia & Propofol and rocuronium & Propofol and rocuronium \\
\hline Opioid & Morphine injection as low as possible. & No restriction of morphine injection use. \\
\hline Monitoring & Hemodynamic parameters. & Hemodynamic parameters. \\
\hline Prophylaxis & Intravenous antibiotic(s). & Intravenous antibiotic(s). \\
\hline Surgery & Laparoscopy/open surgical procedure. & Laparoscopy/open surgical procedure. \\
\hline Warming & Yes. & No. \\
\hline Drains & Minimal use. & Regular use. \\
\hline Fluid infusion & $\leq 1.5 \mathrm{~L}$ & No restriction. \\
\hline Pain management & Epidural analgesia + paracetamol infusion. & Sufentanil. \\
\hline \multirow[t]{4}{*}{ Postoperative diet } & $\begin{array}{l}\text { 1-piece chewing gum three times in } \\
\text { a day (if required). }\end{array}$ & Chewing gum. \\
\hline & $\begin{array}{l}200 \mathrm{~mL} 10 \% \text { glucose within } 1 \text { day after } \\
\text { operation (if required). }\end{array}$ & Fasting until flatus. \\
\hline & $\begin{array}{l}\text { Liquid diet on the next day of operation } \\
\text { (if required). }\end{array}$ & Liquid diet after flatus. \\
\hline & The rehabilitation of diet as early as possible. & Diet after defecation. \\
\hline Intravenous fluid infusion & $\begin{array}{l}\text { Maximum for } 3 \text { days or until nutritional } \\
\text { emulsion administered }\end{array}$ & $\begin{array}{l}\text { High energy fluid on daily basis (infusion) until } \\
\text { oral intake. }\end{array}$ \\
\hline Energy & $25-30$ kcal/kg/day. & $25-30$ kcal/kg/day. \\
\hline Nasogastric tube & Removed after surgery. & Removed after the first flatus. \\
\hline Urethral catheter & Removed within 2 days after surgery. & Removed when automatic micturition feeling. \\
\hline Ambulation & $\begin{array}{l}\text { Within } 24 \mathrm{~h} \text { after surgery, } \geq 1 \mathrm{~h} / \text { day and } \\
\text { gradually increased. }\end{array}$ & No ambulation schemes. \\
\hline $\begin{array}{l}\text { Adjuvant chemotherapy (if required; } \\
\text { institutional protocol) }\end{array}$ & $\begin{array}{l}8 \text { cycles of capecitabine and oxaliplatin; } \\
\text { every } 21 \text { days }\end{array}$ & $\begin{array}{l}12 \text { cycles of leucovorin, fluorouracil, and oxaliplatin; } \\
\text { every } 15 \text { days }\end{array}$ \\
\hline Hospitalization for chemotherapy & 1 day & 3 days \\
\hline
\end{tabular}

All surgical procedures were performed according to the National Comprehensive Cancer Network Clinical Practice Guidelines in Oncology ${ }^{\oplus}$ Colon

Cancer V.2.2018.

during follow-up of patients were retrospectively col- Surgical checkpoints

lected from medical records of the institute and The number of checkpoints to be compliance by the analyzed. planned procedures was noted. There was a maximum 
of 13 checkpoints. If the patient was violated 10 or more checkpoints, then it was considered as the patient was not received planned allocated intervention(s). The checkpoints were (1) preadmission and counseling, (2) surgical preparation, (3) gastrointestinal decompression, (4) perioperative management (general and epidural anesthesia), (5) opioid and prophylaxis administration, (6) hemodynamic parameters monitoring, (7) warming, (8) grains, (9) fluid infusion, (10) Pain management, (11) postoperative diet, (12) removal of nasogastric tube and urethral catheter, and (13) ambulation.

\section{Hospital stays}

Time from hospitalization to discharge was considered as length of hospital stay. Time after the operation to discharge of hospital was considered as postoperative hospital stays. Chemotherapy-related hospital stays were not included in the appropriate groups.

\section{Outcomes for surgery}

Intraoperative blood loss, the readmission within 30 days, surgical cost, self-reported flatus resumption time, the time until the first defecation, resumption of a fluid diet time (time to take nutrition and fluid balance seriously), and ambulation onset time (the time required for the first the act, action, or an instance of moving after surgery) were retrospectively collected from the patients' records of the institute.

\section{Chemotherapy and chemotherapy-related adverse events}

The adjuvant chemotherapy was administered in a later admission after a full recovery, usually 3-4 weeks after post-operative discharge. Patients who had the surgical pathological TNM stage (Tumor, Node, and Metastasis stage; related to AJCC staging system) II, III, or IV have received all cycles of chemotherapy. Patients who ended up being TNM stage I have not received the adjuvant chemotherapy. The National Cancer Institute Common Terminology Criteria for Adverse Events v4.03 was preferred for evaluation of the chemotherapy-related adverse events [16]. The data of the cost of chemotherapy was collected from pharmacy and hospital records of patients.

\section{Quality of life}

The quality of life of patients who received treatment were assessed by the trained instructors (3 years of experiences) of institutes using European Organization for Research and Treatment questionnaires before treatment and after completion of all chemotherapy regimens. The median differences of QLQ-C30/CR38 (quality of life questionnaires-cancer specific 30/colorectal cancer specific 38) score of 10 or more points were considered as clinically significant differences [17].

\section{Statistical analysis}

InStat 3.01 GraphPad, San Diego, USA, was used for statistical analyses purpose. Categorical and ordinal variables are presented as frequency (percentages) and continuous variables are presented as mean \pm standard deviation (SD). Categorical and ordinal variables were analyzed by the chi-square test for independence or Fisher's exact test and continuous variables were analyzed by one-way analysis of variance (ANOVA). The Tukey test (considering critical value $(q)>3.633$ as significant) was used for post hoc analysis. All results were considered significant if $p$ value was reported less than 0.05 .

\section{Results}

\section{Study population}

From 15 January 2018 to 25 December 2019, a total of 611 patients ( $>18$ years old) were pathologically diagnosed as colon or upper rectal (the distance of tumor lower margin from anus $>12 \mathrm{~cm}$ ) cancer and underwent surgery (fast-tract or conventional) followed by chemotherapy at the Hebei Petrochina Central Hospital, Langfang, Heibei, China, and the referring hospitals. Among 611 patients, the tumor of 17 patients was removed by the endoscopic mucosal procedure and 52 patients had faced surgeries followed by chemotherapy of mid-low rectal cancer. Therefore, data of these patients $(n=69)$ were excluded from analysis. Data of 542 patients with colon cancer (preoperative biopsies stage II or stage III) and underwent fast-tack or conventional surgeries (laparoscopy/open surgical procedure) followed by chemotherapy regimens (capecitabine and oxaliplatin or leucovorin, fluorouracil, and oxaliplatin) were included in the analysis. The treatment chart of the included patients is presented in Fig. 1.

\section{Demographic and clinical characteristics}

All patients had the American Society of Anesthesiologists status I or II (except 2 patients) at the time of surgeries. There were no statistical differences for the demographic and clinical characteristics of patients at the time of surgeries ( $p>0.05$ for all, Table 2) among cohorts.

\section{Surgical checkpoints}

A high number of surgical checkpoints were compliance by patient of CSLFO cohort than those of FTLCO $(10.17$ \pm 1.02 /patient vs. $9.13 \pm 1.86 /$ patient, $p<0.0001, q=$ 8.218) and OTLCO (10.17 $\pm 1.02 /$ patient vs. $9.56 \pm 1.67 /$ patient, $p<0.0001, q=4.907)$ cohorts. A high number of surgical checkpoints were compliance by patient of OSLFO cohort than those of FTLCO (10.46 \pm 1.18 / 


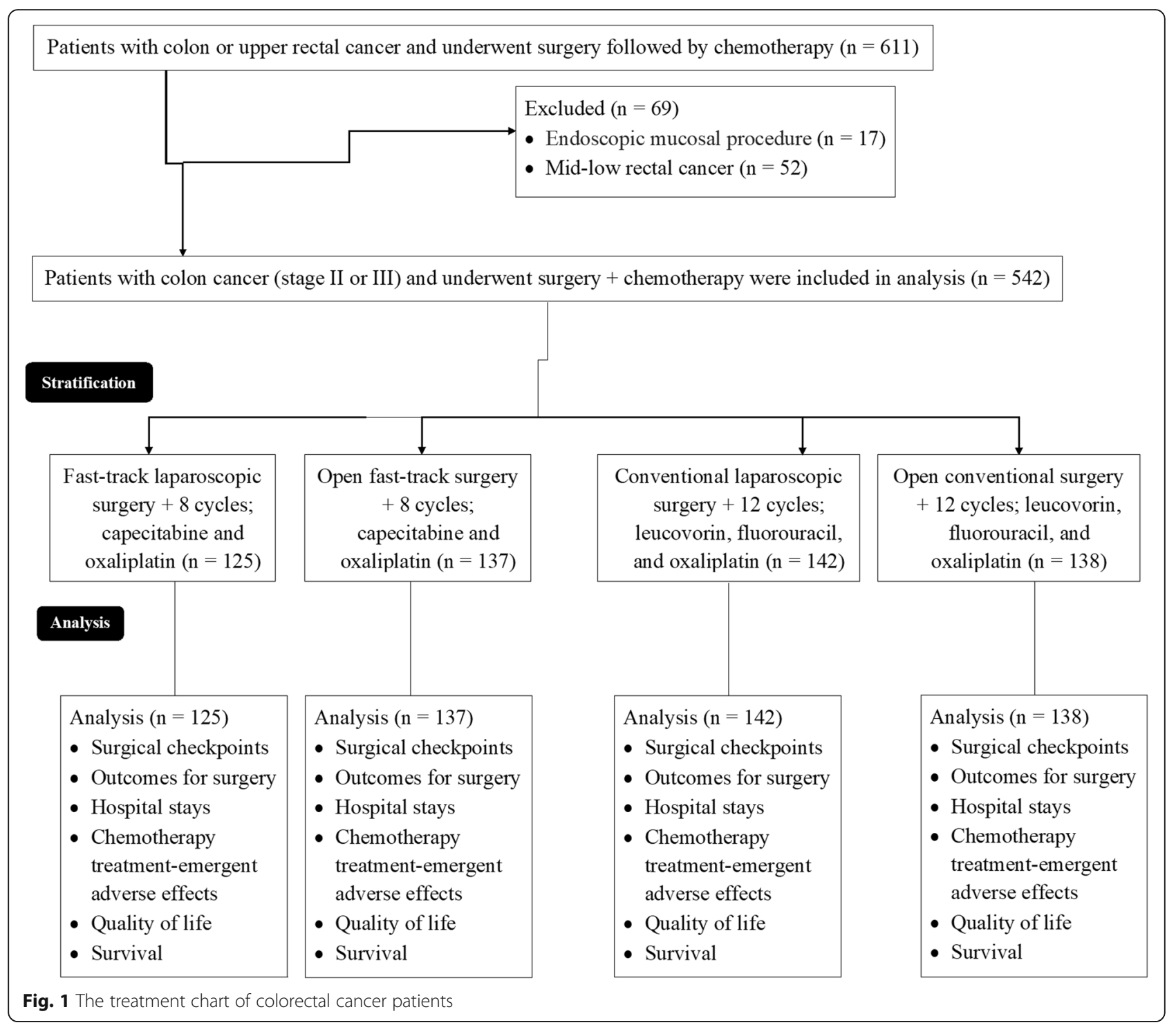

patient vs. $9.13 \pm 1.86 /$ patient, $p<0.0001, q=10.417$ ) and OTLCO (10.46 $\pm 1.18 /$ patient vs. $9.56 \pm 1.67 /$ patient, $p<0.0001, q=7.181)$ cohorts. There was no statistically significant difference for surgical checkpoints compliance/patient between patients of the FTLCO cohort and those of the OTLCO cohort $(p<0.0001, q=$ 3.398). Also, there was no statistically significant difference for surgical checkpoints compliance/patient between patients of the CSLFO cohort and those of the OSLFO cohort $(p<0.0001, q=2.329)$. The details of surgical checkpoints compliance by patients are presented in Fig. 2 .

\section{Hospital stays}

Patients of FTLCO cohort had shorter length of hospital stay than those of CSLFO (14.15 \pm 2.06 days/patient vs. $30.47 \pm 8.37$ days/patient, $p<0.0001, q=27.582)$ and OSLFO (14.15 \pm 2.06 days/patient vs. $34.27 \pm 10.12$ days/ patient, $p<0.0001, q=33.769)$ cohorts. Patients of OTLCO cohort had shorter length of hospital stay than those of CSLFO $(15.67 \pm 2.12$ days/patient vs. $30.47 \pm$ 8.37 days/patient, $p<0.0001, q=25.618)$ and OSLFO (15.67 \pm 2.12 days/patient vs. $34.27 \pm 10.12$ days/patient, $p<0.0001, q=31.962)$ cohorts. There was no statistically significant difference between lengths of hospital stay of patients of FTLCO cohort and those of OTLCO cohort ( $p<0.0001, q=2.253)$. Patients of CSLFO cohort had shorter length of hospital stay than those of OSLFO cohort $(p<0.0001, q=6.583)$.

Patients of FTLCO cohort had shorter postoperative hospital stays than those of CSLFO (7.73 \pm 1.38 days/ patient vs. $10.96 \pm 2.28$ days/patient, $p<0.0001, q=$ $19.223)$ and OSLFO (7.73 \pm 1.38 days/patient vs. 11.42 \pm 2.42 days/patient, $p<0.0001, q=21.829)$ cohorts. Patients of OTLCO cohort had shorter postoperative 


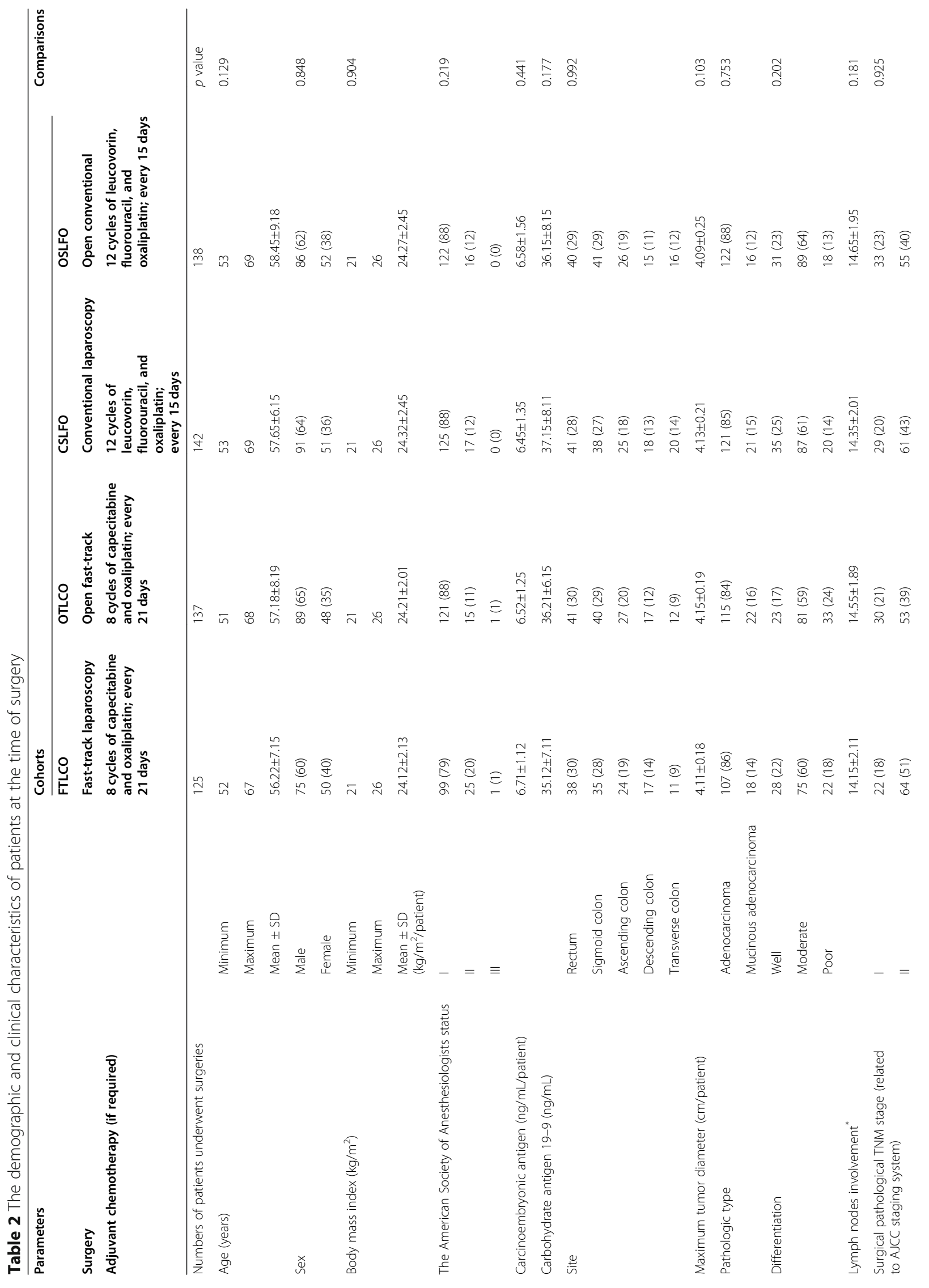




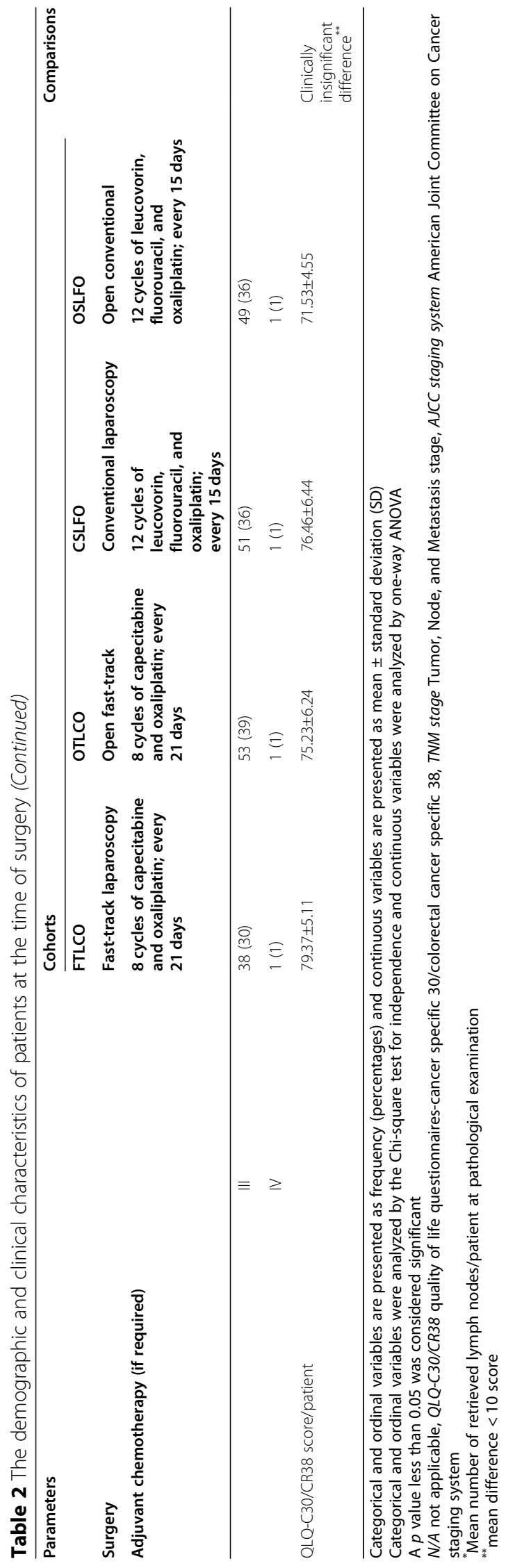




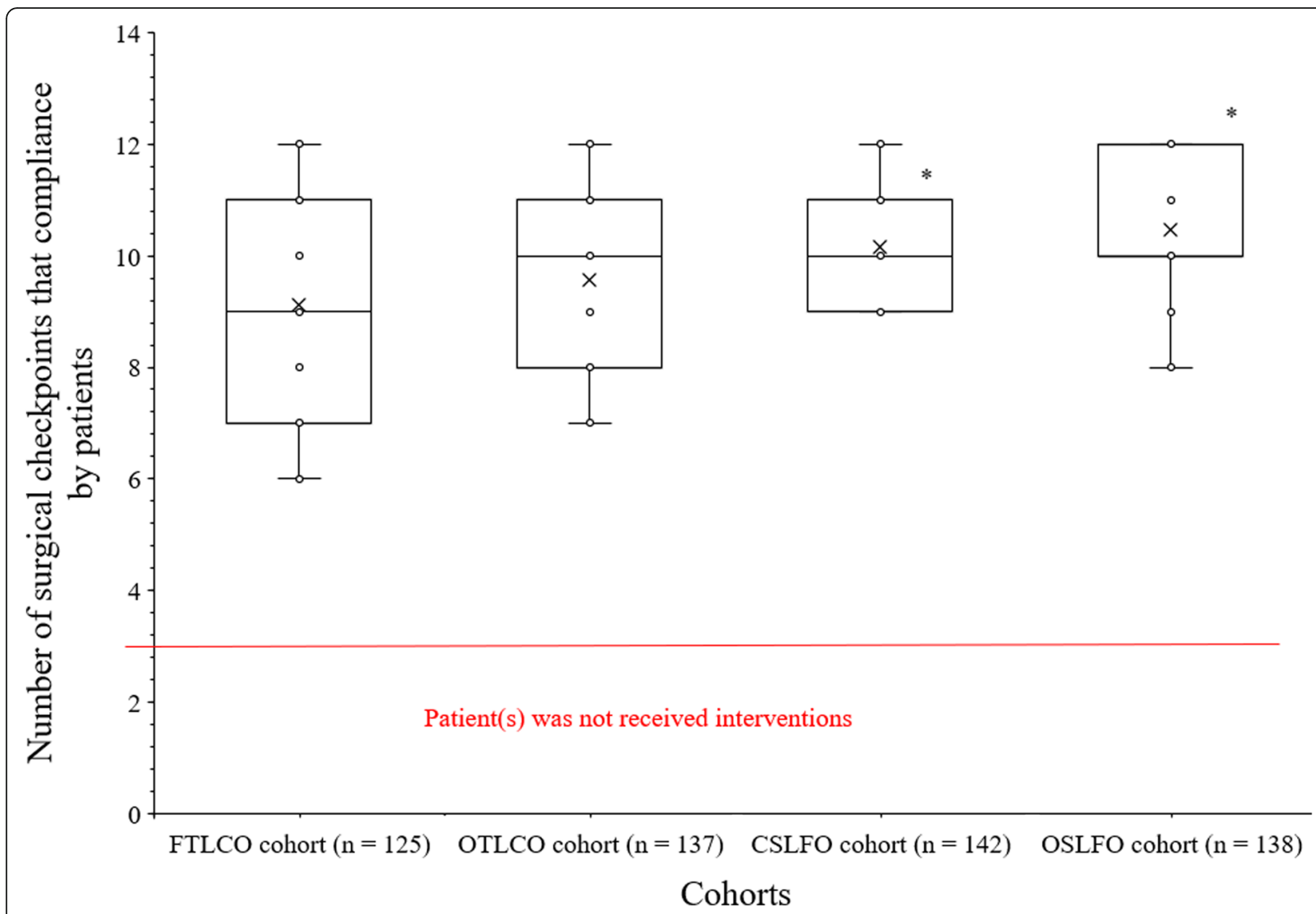

Fig. 2 The number of surgical checkpoints that compliance by patient. Maximum of 13 checkpoints. *Higher number of surgical check-points compliance/patient than those of FTLCO and OTLCO cohorts

hospital stays than those of CSLFO $(8.31 \pm 1.35$ days/ patient vs. $10.96 \pm 2.28$ days/patient, $p<0.0001, q=$ $16.519)$ and OSLFO (8.31 \pm 1.35 days/patient vs. 11.42 \pm 2.42 days/patient, $p<0.0001, q=18.846)$ cohorts. There was no statistically significant difference for postoperative hospital stays between patients of FTLCO cohort and those of OTLCO cohort $(p<0.0001, q=$ 3.145). Also, there was no statistically significant difference for postoperative hospital stays between patients of CSLFO cohort and those of OSLFO cohort $(p<$ $0.0001, q=2.825$ ).

The details of length of hospital stay and postoperative hospital stays are represented in Fig. 3.

\section{Outcomes for surgery}

Flatus resumption time and the time until first defecation were shorter for the patients who underwent fast-track surgical procedures than those who underwent conventional surgical procedures $(p<0.05$ for all). Those postsurgical outcomes were also shorter for patients who underwent the fast-track laparoscopy than those who underwent open fast-track surgical procedure $(p<0.05$ for both). Resumption of a fluid diet time, intraoperative blood loss, and ambulation onset time were shorter for the patients who underwent fast-track surgical procedures than those who underwent conventional surgical procedures $(p<0.05$ for all). Intraoperative blood loss was least for the patients who underwent fast-track laparoscopy surgeries than those who underwent other surgeries $(p<$ $0.05)$. There were no significant differences for the number of the readmission of patients within 30 days among cohorts $(p=0.975)$. The detailed the outcomes for surgery are reported in Table 3.

\section{Surgical cost}

The surgical cost was fewer for patients of OTLCO cohort than those of FTLCO $(30,825 \pm 731 ¥ /$ patient vs. 35,125 \pm $493 ¥ /$ patient, $p<0.0001, q=27.375)$, CSLFO $(30,825 \pm$ $731 ¥ /$ patient vs. $40,475 \pm 2,299 ¥ /$ patient, $p<0.0001, q=$ 63.457), and OSLFO $(30,825 \pm 731 ¥ /$ patient vs. $37,326 \pm$ $2,544 ¥ /$ patient, $p<0.0001, q=42.446)$ cohorts. The surgical cost was fewer for patients of FTLCO than those of CSLFO $(p<0.0001, q=34.353)$ and OSLFO $(p<0.0001, q$ $=14.037)$ cohorts. The surgical cost was fewer for patients of OSLFO cohort than those of CSLFO cohort $(p<$ $0.0001, q=20.748)$. The open fast-track surgeries were 


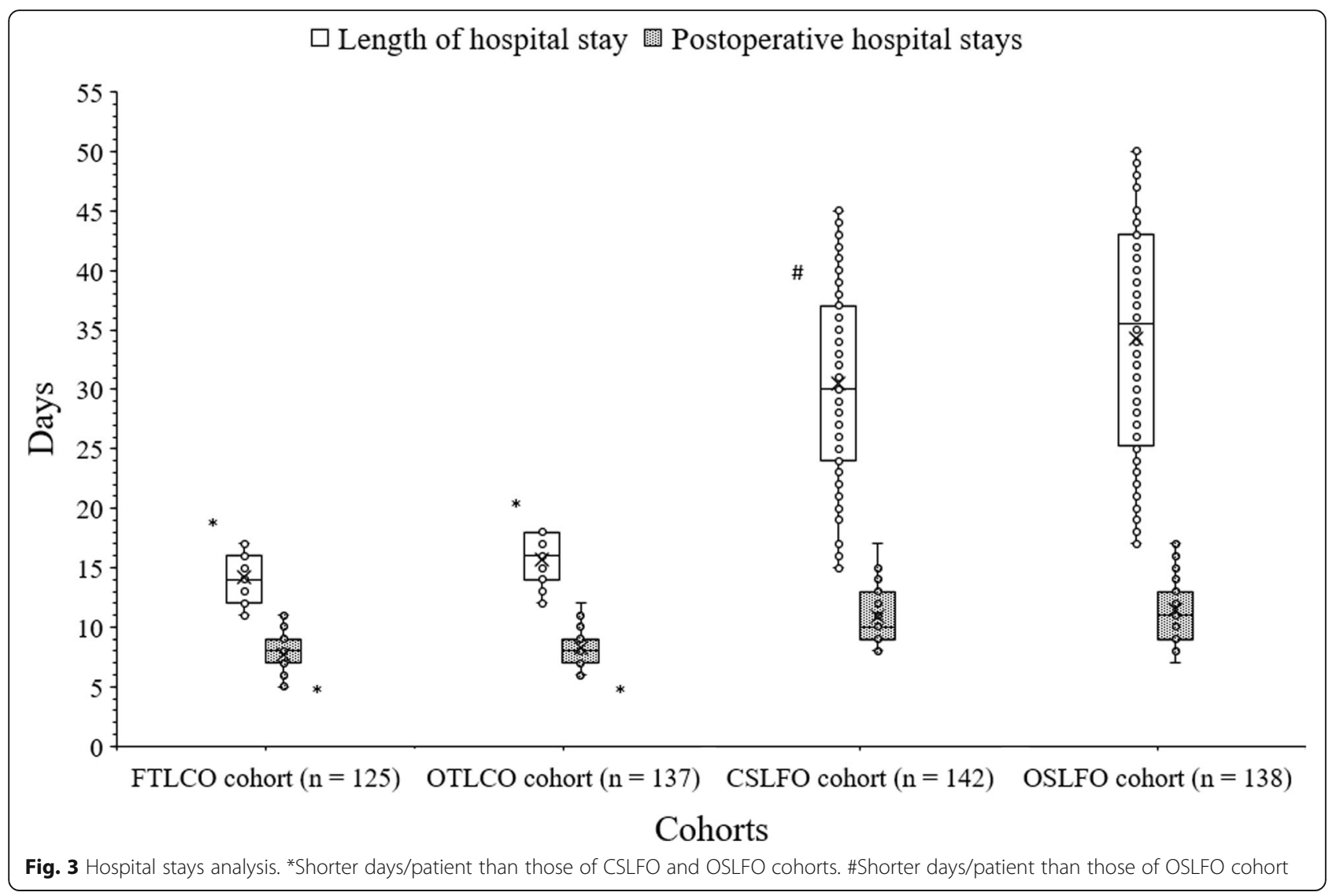

least expensive than the other surgeries. The details of surgical cost are presented in Fig. 4.

\section{Chemotherapy treatment}

A total of 103, 107, 113, and 105 patients of FTLCO cohort, OTLCO cohort, CSLFO cohort, and OSLFO cohort received chemotherapy after post-operative discharge, respectively. There were no significant differences for the number of patients with any grade adverse effects $(p=0.431)$ and the number of patients with grade 3-4 grade adverse effects after chemotherapy $(p=$ $0.858)$. The cost of chemotherapy was statistically the same for patients among cohorts $(p=0.151)$. The details of chemotherapy-emergent adverse effects and the cost of chemotherapy after post-operative discharge are reported in Table 4.

\section{Quality of life}

QLQ-C30/CR38 score was clinically same (mean difference $<10$ score) before surgery among all cohorts. It was clinically lower in case of patients of OSLFO cohort than those of FTLCO cohort after chemotherapy (59.63 \pm 2.26 score/patient vs. $71.67 \pm 5.19$ score/patient, Fig. 5).

\section{Survival}

A total follow-up period was $10-20$ months $(12 \pm 3$ months). Two patients from the OSLFO cohort died during follow-up. The disease-free survival and overall survival were the same for patients among cohorts.

\section{Discussion}

The patients who underwent the fast-track surgical procedures had shorter length of hospital stay and postoperative hospital stays than those who underwent the conventional surgical procedures. The results of the lengths of hospital stay of the current study agreed with those of the LAFA-study [8] and the other available studies on the Chinese population [4, 10-14]. The fasttrack surgical approach is first introduced in the medical oncology for multidiscipline treatments [14]. In the fasttrack surgical approach the colorectal cancer is treated as an integrated disease but not as a surgical disease [4]. The fast-track surgical procedure is effective even in a multidisciplinary scenario as colorectal cancer treatment in which surgery is only a part of management.

The laparoscopic surgical procedures did not reduce the length of hospital stay and postoperative hospital stays than open surgical procedures. Also, the diseasefree survival and overall survival were the same between the fast-track surgical procedures and the conventional 


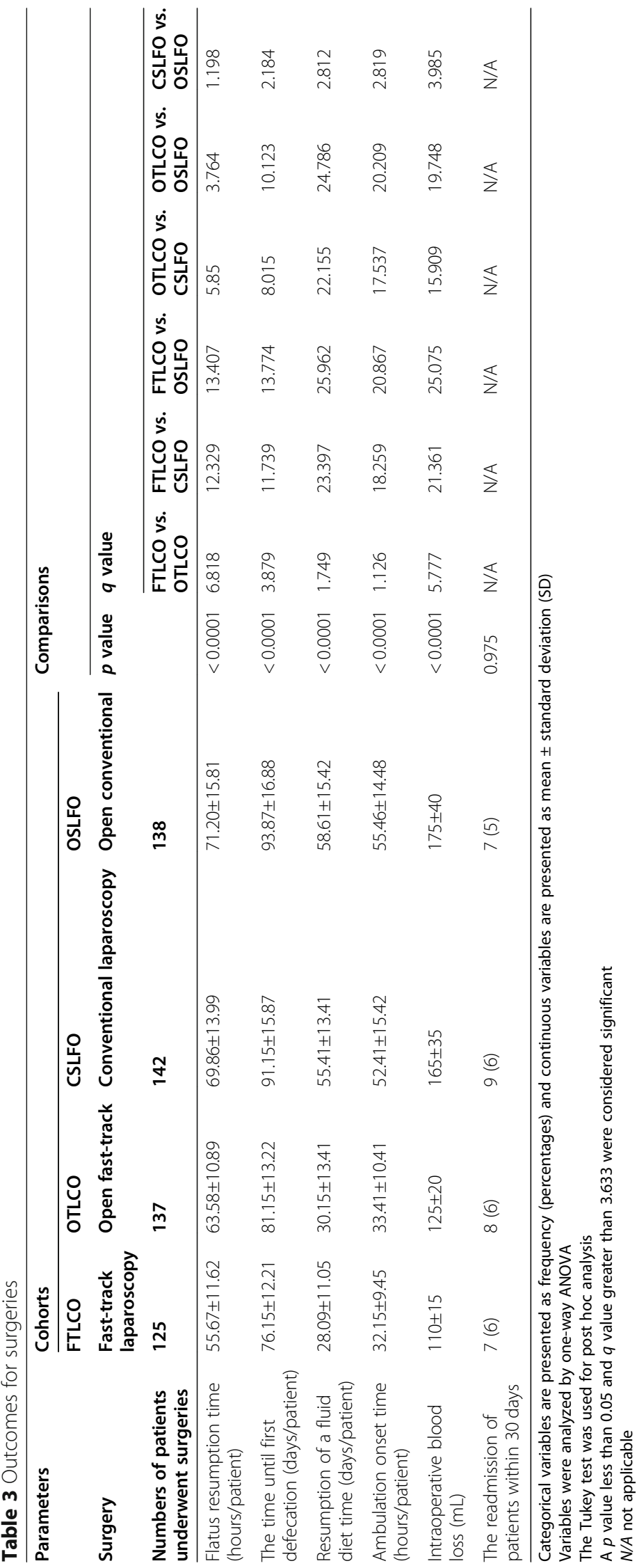




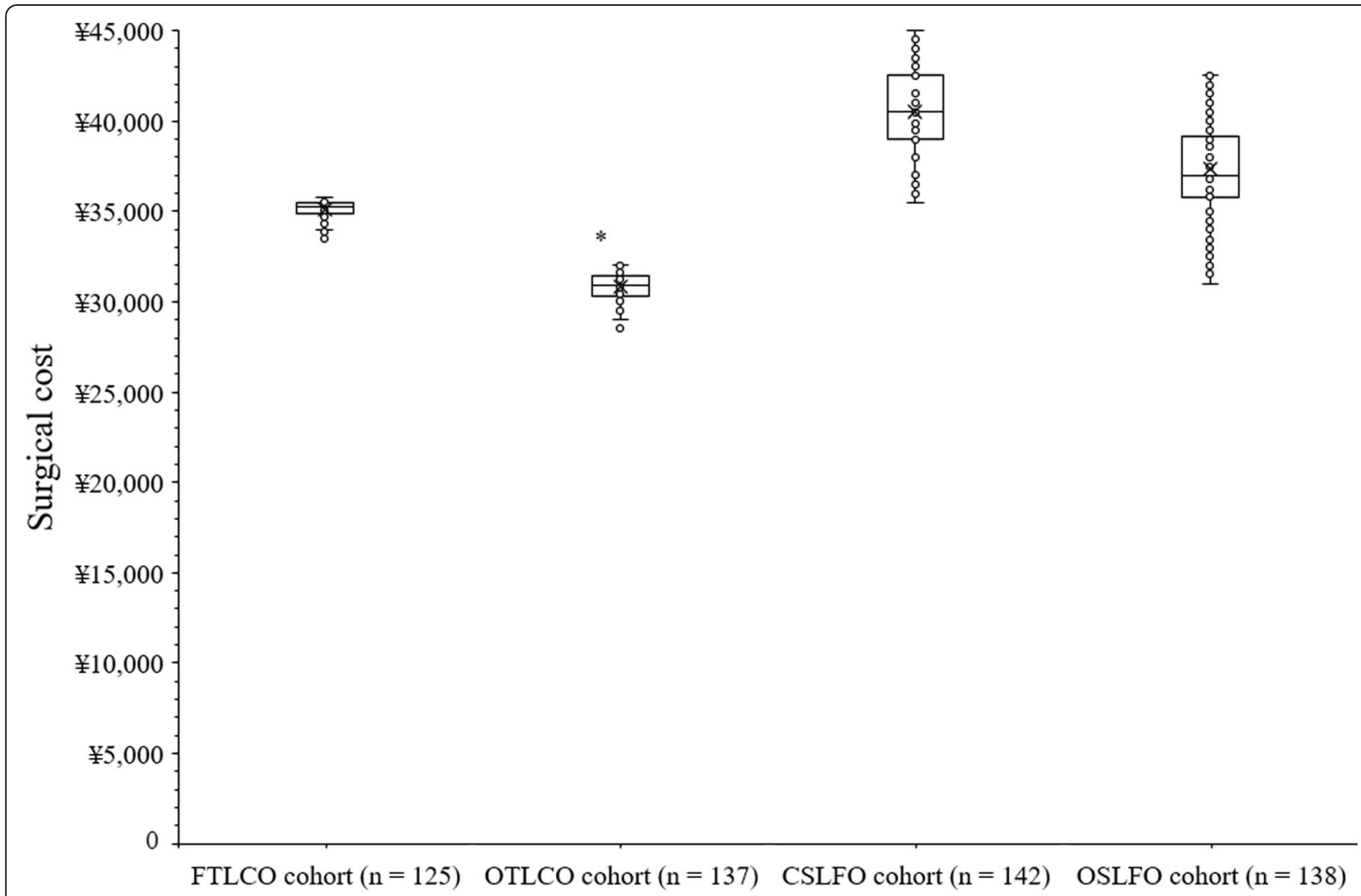

\section{Cohorts}

Fig. 4 Surgical cost of patients. *Fewer $¥ /$ patient than those of FTLCO, CSLFO, and OSLFO cohorts

surgical procedures. The results of the length of hospital stay and survival of the current study were consistent with those of the trial [4]. The fast-track surgical procedures are feasible and have advantages over open surgical procedures for right and left colon cancer [18]. The fast-track surgical approach is as safe as the conventional surgical procedures.

The laparoscopic surgical procedures reduced the flatus resumption time, the time until first defecation, and the intraoperative blood loss. The results of the surgical outcome measures of the current study agreed with those of the other available studies $[4,17]$. During laparoscopies, surgeons efficiently dissect tumors and have high-definition views [4]. The laparoscopic surgical procedures have favorable effects for patients with colorectal cancer than open surgical procedures.

The study is reported that the open fast-track surgeries were least expensive than the other surgeries. The results of the cost of the surgeries of the current study agreed with those of the LAFA-study [8] and the other available study [4]. Thus, the open fast-track surgeries are better choice for colorectal cancer treatment than the conventional laparoscopic surgical procedures or the open conventional surgical procedures.
The study reported that there were no significant differences for the number of patients with any grade adverse effects or with grade 3-4 adverse effects and QLQC30/CR38 score among cohorts. Adjuvant chemotherapy would not affect the quality of the post-operative recovery irrespective of which pathway offered to the patients. The tolerability of chemotherapeutic agent(s) varies greatly from one case to another regardless the kind of surgery or recovery pathway offered to the patients 3-4 weeks before starting chemotherapy. However, patients of FTLCO cohort had clinically better QLQ-C30/CR38 score (changes in 10 or more score) than those of OSLFO cohort after chemotherapy. The justification for the same is that both groups of patients were treated with different chemotherapy regimens.

In the limitation of the study, for example, retrospective study and lack of randomized trial. The surgical checkpoints that were compliance by the patient of fasttrack surgeries were significantly fewer than those of the conventional surgeries. The postoperative hospital stays of patients in the fast-track groups and those of the standard care groups seems to be long for patients with an uncomplicated course. Also, CSLFO and OSLFO patients had statistically similar postoperative hospital 
Table 4 Chemotherapy treatment

\begin{tabular}{|c|c|c|c|c|c|}
\hline \multirow[t]{2}{*}{ Parameters } & \multicolumn{4}{|l|}{ Cohorts } & \multirow[t]{4}{*}{ Comparisons } \\
\hline & FTLCO & OTLCO & CSLFO & OSLFO & \\
\hline Surgery & $\begin{array}{l}\text { Fast-track } \\
\text { laparoscopy }\end{array}$ & Open fast-track & Conventional laparoscopy & Open conventional & \\
\hline $\begin{array}{l}\text { Adjuvant } \\
\text { chemotherapy }\end{array}$ & $\begin{array}{l}8 \text { cycles of } \\
\text { capecitabine and } \\
\text { oxaliplatin; every } 21 \\
\text { days }\end{array}$ & $\begin{array}{l}8 \text { cycles of } \\
\text { capecitabine and } \\
\text { oxaliplatin; every } 21 \\
\text { days }\end{array}$ & $\begin{array}{l}12 \text { cycles of leucovorin, } \\
\text { fluorouracil, and } \\
\text { oxaliplatin; every } 15 \text { days }\end{array}$ & $\begin{array}{l}12 \text { cycles of leucovorin, } \\
\text { fluorouracil, and } \\
\text { oxaliplatin; every } 15 \text { days }\end{array}$ & \\
\hline $\begin{array}{l}\text { Numbers of patients } \\
\text { who received } \\
\text { chemotherapy }\end{array}$ & 103 & 107 & 113 & 105 & $p$ value \\
\hline $\begin{array}{l}\text { Any grade adverse } \\
\text { effects }\end{array}$ & $93(90)$ & $102(95)$ & $107(95)$ & $99(94)$ & 0.431 \\
\hline $\begin{array}{l}\text { Grade } 3-4 \text { adverse } \\
\text { effects }\end{array}$ & $37(36)$ & $39(36)$ & $41(36)$ & $43(41)$ & 0.858 \\
\hline Cost (¥/patient) & $99,000 \pm 10,000$ & $98,500 \pm 17,500$ & $102,000 \pm 15,000$ & $101,500 \pm 10,500$ & 0.151 \\
\hline
\end{tabular}

Categorical and ordinal variables are presented as frequency (percentages) and continuous variables are presented as mean \pm standard deviation (SD) Variables were analyzed by one-way ANOVA

The Tukey test was used for post hoc analysis

A $p$ value less than 0.05 and $q$ value greater than 3.633 were considered significant

The National Cancer Institute Common Terminology Criteria for Adverse Events v4.03 was preferred for evaluation of the chemotherapy-related adverse events

N/A not applicable

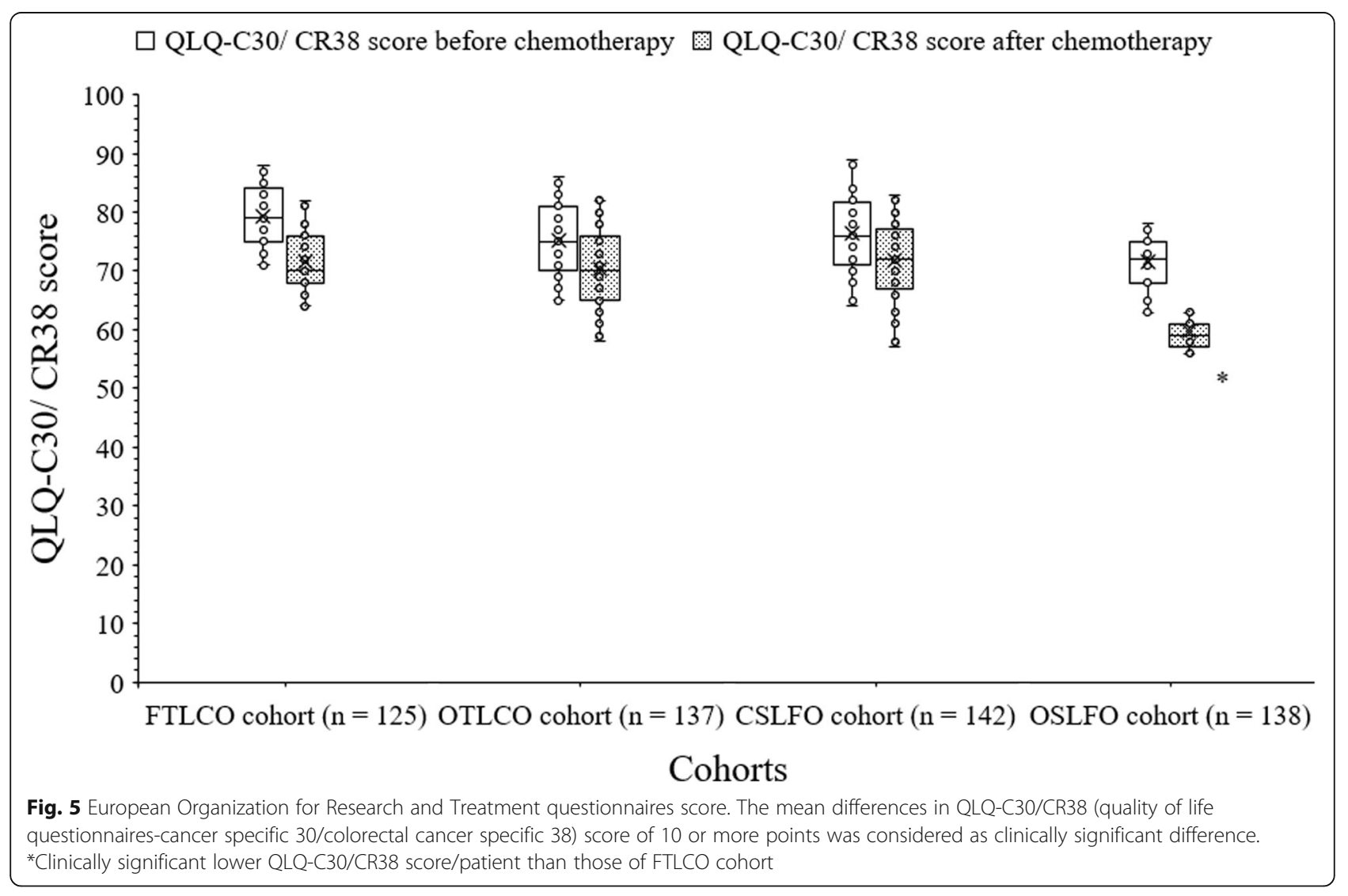


stays. The study did not include the chemotherapyrelated hospital stay in the assessment. The possible justification for the same is the chemotherapy-related hospital stay was fixed ( 3 days for the conventional surgical procedures and 1 day for the fast-track surgical procedures) regardless the kind of the conditions of patients. No information about how patients might have been allocated to the open or laparoscopic procedures and to have or not have fast-track recovery protocols initiated, and how this might have biased the results is not discussed. Chemotherapy dose used as adjuvant treatment in the current study was less than the usually accepted regimens. The disease-free survival and overall survival were the same between the fast-track surgical procedure and the conventional procedure. These conclusions are not entirely correct. A logistic regression could be performed for survival since the groups received different chemotherapy and different surgery but the study lacks a logistic regression analysis.

\section{Conclusions}

The open fast-track surgeries are less expensive. The laparoscopic surgical procedures have favorable effects for patients with colorectal cancer than the open surgical procedures. The fast-track surgical approach is as safe as the conventional surgical procedures and has more favorable effects than the conventional surgical procedures. The study is recommending laparoscopic fasttrack surgical procedures for colorectal cancer treatment. The study reported that there are advantages when adopting early recovery protocols after colorectal cancer surgery, even in patients referred for neoadjuvant chemotherapy treatment. The finding will help the oncologists to provide information about the diffusion of enhanced recovery pathways in China. The study includes high quality and scientifically sound works exploring new surgical technologies and innovative surgical techniques that have the possibility to improve patient care and push the boundaries of surgery.

\section{Abbreviations \\ ANOVA: Analysis of variance; q: The critical value for Tukey test; QLQ-C30/ CR38: Quality of life questionnaires-cancer specific 30/colorectal cancer spe- cific 38; SD: Standard deviation; TNM stage: Tumor, Node, and Metastasis stage; AJCC staging system: American Joint Committee on Cancer staging system}

\section{Acknowledgements}

The authors are grateful to the medical, anesthesiologic, surgical, and nursing staff of the Hebei Petrochina Central Hospital, Langfang, Heibei, China.

\section{Authors' contributions}

Authors declare that this work was done by the authors named in this article and all liabilities pertaining to claims relating to the content of this article will be borne by the authors. All authors have read and approved the manuscript. BW was the project administrator who contributed to the methodology, literature review, data curation, and resources of the study. XW contributed to supervision, methodology, literature review, resources, and validation of the study. RZ contributed to the investigation, methodology, literature review, resources, and formal analysis of the study. YC contributed to the conceptualization, methodology, literature review, resources, and software of the study. JD contributed to data curation, methodology, literature review, resources, and formal analysis of the study. XQ contributed to methodology, literature review, resources, and funding and edited the manuscript for intellectual content. The authors agree to be accountable for all aspects of work ensuring integrity and accuracy.

\section{Funding}

This study was supported by Langfang Science and Technology Support Project of Hebei Province (NO: 2020013089). This funding source had no role in the design of this study and will not have any role during its execution, analyses, interpretation of the data, draft of the manuscript, or decision to submit results.

\section{Availability of data and materials}

The datasets used and analyzed during in this study are available from the corresponding author on reasonable request.

\section{Declarations}

\section{Consent to publication}

None.

\section{Ethics approval and consent to participate}

The designed protocol (2020013089 dated 22 July 2020) was approved by the Hebei Petrochina Central Hospital's review board and the Society of Chinese surgeons. The study reporting adheres to the law of China and the v2008 Declarations of Helsinki. An informed consent form was signed by relatives (legally authorized person) of patients regarding diagnosis, surgery, pathology (if any), chemotherapy, and publication of the anonymized information of patients in the form of the article during hospitalization. As the study was a retrospective analysis, the registration in the Chinese Clinical Trial Registry was waived by the institutional review board.

\section{Competing interests}

The authors declare that they have no competing interests.

Received: 17 January 2021 Accepted: 18 March 2021

Published online: 06 April 2021

\section{References}

1. Siegel RL, Miller KD, Fedewa SA, Ahnen DJ, Meester RGS, Barzi A, et al. Colorectal cancer statistics, 2017. CA Cancer J Clin. 2017;67(3):177-93. https://doi.org/10.3322/caac.21395.

2. Siegel RL, Miller KD, Goding Sauer A, Fedewa SA, Butterly LF, Anderson JC, et al. Colorectal cancer statistics, 2020. CA Cancer J Clin. 2020;70(3):145-64. https://doi.org/10.3322/caac.21601.

3. Feng RM, Zong YN, Cao SM, Xu RH. Current cancer situation in China: Good or bad news from the 2018 Global Cancer Statistics? Cancer Commun. 2018; 39(22):1-12.

4. Li J, Kong XX, Zhou JJ, Song YM, Huang XF, Li GH, Ying XJ, Dai XY, Lu M, Jiang K, Fu DL, Li XL, He JJ, Wang JW, Sun LF, Xu D, Xu JY, Chen M, Tian Y, Li JS, Yan M, Yuan Y, Ding KF. Fast-track multidisciplinary treatment versus conventional treatment for colorectal cancer: a multicenter, open-label randomized controlled study. BMC Cancer 2019; 19: 988-981-10.

5. Vogel JD, Eskicioglu C, Weiser MR, Feingold DL, Steele SR. The American Society of Colon and Rectal Surgeons Clinical Practice Guidelines for the treatment of colon cancer. Dis Colon Rectum. 2017;60(10):999-1117. https:// doi.org/10.1097/DCR.0000000000000926.

6. Gustafsson UO, Scott MJ, Schwenk W, Demartines N, Roulin D, Francis N, et al. Guidelines for perioperative care in elective colonic surgery: Enhanced Recovery After Surgery (ERAS ${ }^{\circledR}$ ) Society recommendations. Clin Nutr. 2012; 31(6):783-800. https://doi.org/10.1016/j.clnu.2012.08.013.

7. Gustafsson UO, Scott MJ, Hubner M, Nygren J, Demartines N, Francis N, et al. Guidelines for Perioperative Care in Elective Colorectal Surgery: Enhanced Recovery After Surgery (ERAS ${ }^{\circ}$ ) Society Recommendations: 2018. World J Surg. 2019;43(3):659-95. https://doi.org/10.1007/s00268-018-4844-y.

8. Vlug MS, Wind J, Hollmann MW, Ubbink DT, Cense HA, Engel AF, et al. Laparoscopy in combination with fast track multimodal management is the 
best perioperative strategy in patients undergoing colonic surgery: a randomized clinical trial (LAFA-study). Ann Surg. 2011;254(6):868-75. https:// doi.org/10.1097/SLA.0b013e31821fd1ce.

9. Kennedy RH, Francis EA, Wharton R, Blazeby JM, Quirke P, West NP, et al. Multicenter randomized controlled trial of conventional versus laparoscopic surgery for colorectal cancer within an enhanced recovery programme: EnROL. J Clin Oncol. 2014;32(17):1804-11. https://doi.org/10.1200/JCO.2013. 54.3694.

10. Feng F, Li XH, Shi H, Wu GS, Zhang HW, Liu XN, et al. Fast-track surgery combined with laparoscopy could improve postoperative recovery of lowrisk rectal cancer patients: a randomized controlled clinical trial. J Dig Dis. 2014;15(6):306-13. https://doi.org/10.1111/1751-2980.12142.

11. Ren L, Zhu D, Wei Y, Pan X, Liang L, Xu J, et al. Enhanced Recovery After Surgery (ERAS) program attenuates stress and accelerates recovery in patients after radical resection for colorectal cancer: a prospective randomized controlled trial. World J Surg. 2012;36(2):407-14. https://doi. org/10.1007/s00268-011-1348-4.

12. Wang G, Jiang ZW, Zhao K, Gao Y, Liu FT, Pan HF, et al. Fast track rehabilitation programme enhances functional recovery after laparoscopic colonic resection. Hepatogastroenterology. 2012;59(119):2158-63. https:// doi.org/10.5754/hge11957.

13. Yang D, He W, Zhang S, Chen H, Zhang C, He Y. Fast-track surgery improves postoperative clinical recovery and immunity after elective surgery for colorectal carcinoma: randomized controlled clinical trial. World J Surg. 2012;36(8):1874-80. https://doi.org/10.1007/s00268-012-1606-0.

14. Zhou JJ, Li J, Ying XJ, Song YM, Chen R, Chen G, Yan M, Ding KF. Fast track multi-discipline treatment (FTMDT trial) versus conventional treatment in colorectal cancer--the design of a prospective randomized controlled study. BMC Cancer 2011; 11: 494-1-10.

15. Benson AB, Venook AP, Al-Hawary MM, Cederquist L, Chen YJ, Ciombor KK et al. NCCN Guidelines Insights: Colon Cancer, Version 2.2018. J Natl Compr Canc Netw. 2018;16:359-69.

16. US Department of Health and Human Services. Common Terminology Criteria for Adverse Events (CTCAE) v4.03. Available from: https://evs.nci.nih. gov/ftp1/CTCAE/CTCAE_4.03/CTCAE_4.03_2010-06-14_QuickReference_8. 5x11.pdf. [15 January 2017].

17. Li J, Chen $R$, Xu YQ, Wang XC, Zheng S, Zhang SZ, et al. Impact of a laparoscopic resection on the quality of life in rectal cancer patients: results of 135 patients. Surg Today. 2010;40(10):917-22. https://doi.org/10.1007/ s00595-009-4156-9.

18. Gavriilidis P, Davies RJ, Biondi A, Wheeler J, Testini M, Carcano G, et al. Laparoscopic versus open complete mesocolic excision: a systematic review by updated meta-analysis. Updates Surg. 2020;72(3):639-48. https://doi. org/10.1007/s13304-020-00819-1.

\section{Publisher's Note}

Springer Nature remains neutral with regard to jurisdictional claims in published maps and institutional affiliations.

Ready to submit your research? Choose BMC and benefit from:

- fast, convenient online submission

- thorough peer review by experienced researchers in your field

- rapid publication on acceptance

- support for research data, including large and complex data types

- gold Open Access which fosters wider collaboration and increased citations

- maximum visibility for your research: over $100 \mathrm{M}$ website views per year

At $\mathrm{BMC}$, research is always in progress.

Learn more biomedcentral.com/submissions 